\title{
ON SOME CLASSES OF SPECTRAL POSETS
}

\author{
By
}

\author{
Tomoo YokoYama
}

\begin{abstract}
This paper deals with sufficient conditions on a poset in order to get it spectral. A motivating question is the following (p. 833 [LO76]): "If $X$ is a height 1 poset such that for all $x \neq y \in X, \uparrow x \cap \uparrow y$ and $\downarrow x \cap \downarrow y$ are finite, is $X$ spectral?" We obtain the some sufficient conditions for such a poset $X$ to be spectral. In particular, we prove that either if there is a finite subset $F \subseteq X$ such that $\downarrow F \supseteq \operatorname{Min} X$, or if diam $X \leq 2$, then the poset $X$ is spectral.
\end{abstract}

\section{Introduction and Preliminaries}

W. J. Lewis and J. Ohm showed the following result [LO76]: An ordered disjoint union $X$ of spectral posets $\left(X_{\lambda}\right), \lambda \in \Lambda$ is spectral. In the same paper, they also showed that if a height 1 poset $X$ satisfies that for all $x \in X, \uparrow x \cap \uparrow y=\varnothing$ and $\downarrow x \cap \downarrow y=\varnothing$ for all but finite many $y \in X$, then $X$ is spectral. Moreover, they asked the following analogous two questions: (1) If a spectral poset $X$ is the ordered disjoint union of posets $\left(X_{\lambda}\right), \lambda \in \Lambda$, are the $X_{\lambda}$ also spectral? (2) If a height 1 poset $X$ satisfies that for all $x \neq y \in X, \uparrow x \cap \uparrow y$ and $\downarrow x \cap \downarrow y$ are finite, is $X$ spectral? In [BE04], Belaid and Echi studied the both question. For the second question, several authors contributed to the question (e.g. [BF81], [DFP80], [F79], and [LO76]). The first question was answered negatively in [AZ04]. In particular, M. E. Adams and van der Zypen constructed a negative example (i.e., an example which is not a spectral poset but can be embedded in some spectral poset). Note that there is a non-spectral poset which can not be embedded as a connected component in any spectral poset (see Example 3.3). On the other hand, the second was also answered negatively in [Y09]. In particular, one showed that there are height 1 countable non-spectral posets $X$ with diameter $\geq 3$ such that

2000 Mathematics Subject Classification. Primary: 06A06; Secondary: 54F05.

Key words and phrases. spectral posets, height 1 posets.

Received May 6, 2010.

Revised August 25, 2010. 
for all $x \neq y \in X, \uparrow x \cap \uparrow y$ and $\downarrow x \cap \downarrow y$ are finite subsets. In contrast, we consider the sufficient conditions for a height 1 poset to be spectral, which are similar to the condition in the second question.

Recall that a poset $(X, \leq)$ is said to be spectral or representable if there is a commutative ring $R$ with unit such that $X$ is order isomorphic to the set $\operatorname{Spec}(R)$ of its prime ideals with the inclusion order. Define the height of $X$ is the supremum of lengths of chains in $X$. For an element $x$ of a poset $X$, $\uparrow x:=\{y \in X \mid x \leq y\}$ and $\downarrow x:=\{y \in X \mid y \leq x\}$ are called the saturation of $x$ and the cosaturation of $x$ respectively. Note that $\uparrow x$ (resp. $\downarrow x)$ is also called the set of generalization (resp. specialization) of $x$.

For a subset $Y \subseteq X, \uparrow Y:=\bigcup_{y \in Y} \uparrow y$ and $\downarrow Y:=\bigcup_{y \in Y} \downarrow y$ are called the saturation of $Y$ and the cosaturation of $Y$ respectively. A subset $Y \subseteq X$ is called a saturation or a upset if $Y=\uparrow Y$. Similarly a subset $Y \subset X$ is also called a cosaturation or a downset if $Y=\downarrow Y$.

Define the diameter diam $X$ of a poset $X$ as the minimal number $n$ such that there is $x \in X$ such that either $(\uparrow \downarrow)^{k} x=X$ or $(\downarrow \uparrow)^{k} x=X$ whenever $n=2 k$ is even, and either $(\uparrow \downarrow)^{k} \uparrow x=X$ or $\downarrow(\uparrow \downarrow)^{k} x=X$ whenever $n=2 k+1$ is odd. Here, by induction, we mean that $(\uparrow \downarrow) x=\uparrow(\downarrow x)=\{y \in X \mid y \in \uparrow z$ for some $z \in \downarrow x\}$, $\downarrow(\uparrow \downarrow) x=\downarrow(\uparrow(\downarrow x))=\{y \in X \mid y \in \downarrow z$ for some $z \in \uparrow \downarrow x\},(\uparrow \downarrow)^{2} x=\uparrow(\downarrow(\uparrow(\downarrow x)))$, and so on. In general, $(\downarrow \uparrow)^{k} x$ and $(\uparrow \downarrow)^{k} x$ are different even if $k=1$ and the height of $X$ is one.

For a subset $Y \subseteq X$, denote by $\operatorname{Min} Y$ (resp. Max $Y$ ) the set of minimal (resp. maximal) elements of $Y$ with respect to the restricted order. The connected component or the order component of $X$ containing an element $x \in X$ is the subset $S$ of $X$ of all elements $y$ which have a path $y=y_{0} \leq y_{1} \geq y_{2} \leq \cdots \geq x$ from $y$ to $x$. If $X$ has only one component, then $X$ is said to be connected.

A topological space $X$ is said to be spectral if there is a commutative ring $R$ with unit such that $X$ is homeomorphic to the $\operatorname{set} \operatorname{Spec}(R)$ of its prime ideals with the Zariski topology.

In [H69], Hochster showed that a topological space $X$ is spectral if and only if $X$ is $T_{0}$, sober and compact, and has a compact open basis closed under finite intersections.

Let $(X, T)$ be a topological space and $\leq$ a partial order on $X$. The topology $T$ is said to be order compatible with $\leq$, if $\overline{\{x\}}=\downarrow x$, for each $x \in X$. One can obviously see that $(X, \leq)$ is spectral if and only if there exists an order compatible spectral topology on $X$.

A poset $(X, \leq)$ with an order compatible topology is called a CTOD (or Priestley) space if $X$ is compact and is totally order-disconnected in the sense 
that, given $y \not \leq x \in X$, there exists a clopen downset $U$ such that $x \in U, y \notin U$. By the results in [S37] and [P94], it is shown that a poset $X$ is spectral if and only if $X$ has a CTOD-topology. Note that a poset $(X, \leq)$ is spectral if and only if the poset $(X, \geq)$ with the opposite order is spectral.

We obtain the following result, which is a generalization of Corollary (p. 166 [BF81]).

THEOREM 1.1. Let $(X, \leq)$ be a height 1 connected poset. Suppose that $|\downarrow x \cap \downarrow y|<\infty$ for any elements $x \neq y$ of $X$. If there is a finite subset $F \subseteq X$ such that $\downarrow F \supseteq \operatorname{Min} X$, then $X$ is a spectral poset. In particular, if either Max $X$ or Min $X$ is finite, then $X$ is spectral.

By the well-known fact that for a spectral poset $(X, \leq)$ the set $(X, \geq)$ with the reverse order is spectral, the dual statement of the above result holds.

Because any height 1 poset $X$ with diameter $\leq 2$ has an element $x \in X$ such that either $\uparrow x \supseteq \operatorname{Max} X$ or $\downarrow x \supseteq \operatorname{Min} X$, the poset $X$ satisfies the conditions in the above theorem or the dual statement. The following corollary is induced.

COROllary 1.2. Any height 1 poset $X$ with diameter $\leq 2$ and with $|\uparrow x \cap \uparrow y|+|\downarrow x \cap \downarrow y|<\infty$ for any distinct elements $x \neq y \in X$ is spectral.

This result is in stark contrast to the existence of non-spectral height 1 poset with diameter 3 satisfying the finiteness condition in the above corollary. We will show the following corollary in the next section.

COROLlary 1.3. Let $(X, \leq)$ be a height 1 poset with connected components $X_{i}, i \in I$. Suppose that $|\downarrow x \cap \downarrow y|<\infty$ for any elements $x \neq y$ of $X$. If there are finite subsets $F_{i} \subseteq X$ for all $i \in I$ such that $\bigcup_{i \in I} \downarrow F_{i} \supseteq\{x \in X:|\downarrow x|+|\uparrow x|=\infty\}$, then $X$ is spectral.

\section{Proofs of Results}

In this section, we show Theorem 1.1 and Corollary 1.3.

Proof of TheOrem 1.1. Let $w_{1}, \ldots, w_{n}$ be finitely many elements of $X$ such that $\bigcup_{i=1}^{n} \downarrow w_{i} \supseteq \operatorname{Min} X$. Let $Y=X-\bigcup_{i=1}^{n} \downarrow w_{i}=\operatorname{Max} X-\left\{w_{1}, \ldots, w_{n}\right\}$. Since $\downarrow y \cap \downarrow w_{i}$ for any $y \in Y$ and any $i=1, \ldots, n$ is finite, this implies that $\downarrow y \cap \operatorname{Min} X=\bigcup_{i=i}^{n}\left(\downarrow y \cap \downarrow w_{i}\right)$ is finite. Thus $\downarrow y$ is finite for any element $y \in Y$. Let 
$W=\bigcup_{i \neq j} \downarrow w_{i} \cap \downarrow w_{j}$. Since any intersection of cosaturation of two distinct elements is finite, $W$ is finite. Define an order compatible topology $T$ of $X$ by the closed subbasis $\mathscr{F}_{X}=\{\downarrow F: F \subseteq X$ is finite $\} \cup\{X-S: S \subseteq Y\}$.

ClaIm 2.1. $\mathscr{F}_{X}$ is the set of all closed subsets.

Indeed, put $\mathscr{F}_{0}:=\{\downarrow F: F \subseteq X$ is finite $\}$ and $\mathscr{F}_{1}:=\bigcup\{X-S: S \subseteq Y\}$. For $C \in \mathscr{F}_{0}$, there are $L \subseteq\{1, \ldots, n\}$ and a finite downset $F \subseteq X-\left\{w_{1}, \ldots, w_{n}\right\}$ such that $C=\bigcup_{i \in L} \downarrow w_{i} \cup F$. For $C_{1}, \ldots, C_{n} \in \mathscr{F}_{X}$, if there is $i \in\{1, \ldots, n\}$ such that $C_{i} \in \mathscr{F}_{1}$, then $\bigcup_{i=1}^{n} C_{i} \in \mathscr{F}_{1}$. Otherwise $C_{1}, \ldots, C_{n} \in \mathscr{F}_{0}$ and so there are $L \subseteq\{1, \ldots, n\}$ and a finite downset $F \subseteq X-\left\{w_{1}, \ldots, w_{n}\right\}$ such that $\bigcup_{i=1}^{n} C_{i}=$ $\bigcup_{i \in L} \downarrow w_{i} \cup F \in \mathscr{F}_{0}$. Thus $\mathscr{F}_{X}$ is closed under finite unions. Therefore it suffices to show that $\mathscr{F}_{X}$ is closed under arbitrary intersections. For $\left\{C_{\lambda}\right\}_{\lambda \in \Lambda} \subseteq \mathscr{F}_{X}$, if $\left\{C_{\lambda}\right\}_{\lambda \in \Lambda} \subseteq \mathscr{F}_{1}$ then $\bigcap_{\lambda \in \Lambda} C_{\lambda} \in \mathscr{F}_{1}$. Replacing $\left\{C_{\lambda}\right\}_{\lambda \in \Lambda} \cap \mathscr{F}_{0}$ by $\bigcap\left\{C_{\lambda} \mid C_{\lambda} \in \mathscr{F}_{0}\right.$, $\lambda \in \Lambda\}$, we may assume that $\left|\left\{C_{\lambda}\right\}_{\lambda \in \Lambda} \cap \mathscr{F}_{1}\right| \leq 1$. If there is a unique element $C \in \mathscr{F}_{1}$, then either $\left\{C_{\lambda}\right\}_{\lambda \in \Lambda}$ consists of exactly a single element $C$ or there is some $C_{\lambda} \in \mathscr{F}_{0} \cap\left\{C_{\lambda}\right\}_{\lambda \in \Lambda}$. Thus we may assume that there is some $C_{\lambda} \in \mathscr{F}_{0} \cap\left\{C_{\lambda}\right\}_{\lambda \in \Lambda}$. Then there are $L \subseteq\{1, \ldots, n\}$ and a finite downset $F \subseteq X-\left\{w_{1}, \ldots, w_{n}\right\}$ such that $C \cap C_{\lambda}=\bigcup_{i \in L} \downarrow w_{i} \cup F \in \mathscr{F}_{0}$. Replacing $C$ by $C \cap C_{\lambda}$, we may assume that $\left\{C_{\lambda}\right\}_{\lambda \in \Lambda} \subseteq \mathscr{F}_{0}$. Since each intersection $\downarrow x \cap \downarrow x^{\prime}$ for any distinct elements $x \neq$ $x^{\prime} \in X$ is finite, by the forms of elements of $\mathscr{F}_{0}$, there are $L \subseteq\{1, \ldots, n\}$ and a finite downset $F \subseteq X-\left\{w_{1}, \ldots, w_{n}\right\}$ such that $\bigcap_{\lambda \in \Lambda} C_{\lambda}=\bigcup_{i \in L} \downarrow w_{i} \cup F \in \mathscr{F}_{0}$. Thus $\mathscr{F}_{X}$ is closed under arbitrary intersections.

For $L \subseteq\{1, \ldots, n\}$, denote $U_{L}=X-\bigcup_{i \in L} \downarrow w_{i}$. Then there is an open basis $\mathscr{B}=\mathscr{B}_{0} \cup \mathscr{B}_{1}$, where $\mathscr{B}_{0}=\left\{V \cap U_{L}: V\right.$ is a cofinite upset in $\left.X, L \subseteq\{1, \ldots, n\}\right\}$, $\mathscr{B}_{1}=\{U \subseteq Y$ : finite $\}$. Notice that $\mathscr{B}_{0}=\left\{X-C \mid C \in \mathscr{F}_{0}\right\}$ and $\mathscr{B}_{1}=\{X-C \mid$ $\left.C \in \mathscr{F}_{1}\right\}$. Hence $\mathscr{B}$ is the set of all open subsets. We will show that $\mathscr{B}$ consists of compact subsets. It suffices to show the following claim:

Claim 2.2. For $L \subseteq\{1, \ldots, n\}$ and a cofinite upset $V \subseteq X$, the open subset $U=V \backslash \bigcup_{i \in L} \downarrow w_{i}$ is compact.

Indeed, let $L_{i}=\{1, \ldots, n\}-\{i\}$. Since $U_{L} \subseteq Y \cup \bigcup_{i \notin L} \downarrow w_{i}, \quad Y \subseteq U_{L_{i}}$, and $\downarrow w_{i} \backslash W \subseteq U_{L_{i}}$, these imply that $U_{L} \backslash W \subseteq \bigcup_{i \notin L} U_{L_{i}}$. Since $U_{L} \supseteq \bigcup_{i \notin L} U_{L_{i}}$ and $W$ is finite, we have that $U_{L} \backslash W$ is cofinite in $\bigcup_{i \notin L} U_{L_{i}}$. Let $U$ as in Claim 2.2. Since $U^{\prime}:=U \backslash W \subseteq U_{L} \backslash W$ is open and cofinite in $\bigcup_{i \notin L} U_{L_{i}}$, the finiteness of $W$ implies that $U^{\prime} \cap U_{L_{i}}$ is cofinite in $U_{L_{i}}$ for any $i \notin L$. Since all nonempty open subset in $U_{L_{i}}$ is cofinite in $U_{L_{i}}$, we obtain that $U^{\prime} \cap U_{L_{i}}$ is compact for any $i \notin L$. 
Hence $U^{\prime}=\bigcup_{i \notin L}\left(U^{\prime} \cap U_{L_{i}}\right)$ is compact. Since $W$ is finite, $U=U^{\prime} \cup(U \cap W)$ is compact.

In particular, Claim 2.2 implies that $X$ is compact. Therefore the following claim completes this proof.

Claim 2.3. $X$ is sober.

Indeed, let $F$ be a closed subset. Then $F$ is either a cosaturation $F=\bigcup_{i=1}^{l} \downarrow x_{i}$ of a finite subset or $F=X-S$ where $S \subseteq Y$ is a upset. It suffices to show that $F$ is reducible or has a generic point. Therefore we may assume that $F=X-S$. If $S \neq Y$, then there is an element $x \in Y \backslash S \subset \operatorname{Max} X$ such that $\downarrow x \subseteq F$ and $F-x=X-(\{x\} \sqcup S)$ are closed. Thus $F$ is reducible or $\downarrow x=F$. Otherwise $S=Y$. Then $F=\bigcup_{i=1}^{n} \downarrow w_{i}$. If $n=1$, then $F$ has a generic point $w_{1}$. Otherwise $F$ is reducible.

Proof of Corollary 1.3. Since any ordered disjoint union of spectral posets is spectral, we may assume that $X$ is connected. Suppose that there is a finite subset $\left\{w_{1}, \ldots, w_{n}\right\} \subseteq X$ such that $\bigcup_{i=1}^{n} \downarrow w_{i} \supseteq\{x \in X:|\uparrow x|+|\downarrow x|=\infty\}$. Let $Z=\bigcup_{i=1}^{n} \uparrow \downarrow w_{i}$ and $Y=\operatorname{Min} X \backslash Z$. Notice that for any $y \in Y,|\uparrow y|+|\downarrow y|<\infty$. Since $\bigcup_{i=1}^{n} \downarrow w_{i} \supseteq \operatorname{Min} Z$, Theorem 1.1 implies that $Z$ is a spectral poset. Since $\operatorname{Max} X \backslash Z$ has height 0 and so is a spectral poset, the order disjoint union $Z^{\prime}:=(\operatorname{Max} X \backslash Z) \sqcup Z$ is a spectral poset. Note that $Y$ is a downset and $X=Z^{\prime} \sqcup Y$. To apply Theorem 5.8 [LO76] to $X_{1}=Y$ and $X_{2}=Z^{\prime}$, it is enough to show that, for any $x \in Z^{\prime}$ and for any $y \in Y, \downarrow x \cap Y$ and $\uparrow y \cap Z^{\prime}$ are finite. For $x \in Z$, the definition of $Z$ implies that $\downarrow x \cap Y$ is finite. For $x \in Z^{\prime}-Z$, $|\downarrow x \cap Y| \leq|\downarrow x|<\infty$. For any $y \in Y,\left|\uparrow y \cap Z^{\prime}\right| \leq|\uparrow y|<\infty$. Hence Theorem 5.8 [LO76] implies that $X$ is spectral.

\section{Examples}

We describe some spectral posets.

EXAmPle 3.1. Let $X_{0}=\left\{c_{i}\right\}_{i \in \mathbf{Z}_{>0}} \cup\{w\}$ be a set and $X_{1}=\left\{b_{i}\right\}_{i \in \mathbf{Z}_{>0}} \cup\{a\}$ a set. Define a poset $X=X_{0} \sqcup X_{1}$ with an order $\leq$ as follows: $c_{i}<a, w<b_{i}$ and $c_{i}<b_{i}$ for any $i$. Then Theorem 1.1 implies that $X$ is spectral.

Example 3.2. Let $X$ as in Example 3.1. Define a poset $Y=X \sqcup\left\{w_{i}\right\}_{i \in \mathbf{Z}_{>0}}$ with an extension order $\leq_{Y}$ of $\leq$ by $w, w_{2}<_{Y} w_{1}$ and $w_{2 i}, w_{2 i+2}<_{Y} w_{2 i+1}$ for any $i \in \mathbf{Z}_{>0}$. Then Corollary 1.3 implies that $X$ is spectral. 
The following example is a non-spectral poset which can not be embedded as a connected component in any spectral poset. Recall that the topology on a poset $X$ which is generated by the closed base $\{\downarrow F \mid F \subseteq X$ is finite $\}$ is called the upper topology on $X$.

Example 3.3. Let $X_{0}=\mathbf{Z}_{>1}$ and $X_{1}=\operatorname{Spec} \mathbf{Z}-\{(0)\}=\{(2),(3),(5), \ldots\}$. For $n \in \mathbf{Z}_{>1}$, define $X_{1 n}:=\left\{(p) \in X_{1} \mid p \leq n\right\}$. Define a poset $X_{n}=X_{0} \sqcup X_{1 n}$ with an order $\leq$ as follows: $m<(p)$ if and only if $m / p \in \mathbf{Z}$. Then the dual statement of Corollary 1.3 implies that $X_{n}$ is spectral. However the colimit $X=X_{0} \sqcup X_{1}$ of $X_{n}$ is not spectral and can not be embedded as a connected component in any spectral poset. Indeed, since $\bigcap_{(p) \in X_{1}} \downarrow(p)=\varnothing, \downarrow(p)$ is closed but not compact with respect to the upper topology. Thus $X$ is not compact with respect to the upper topology. Since any order compatible spectral topology contains the upper topology, $X$ can not be embedded as a connected component in any spectral poset.

The following example which is a non-spectral poset $X$ with diameter 2 shows that the finiteness condition (i.e. $|\downarrow x \cap \downarrow y|<\infty$ for any elements $x \neq y \in X$ ) in Theorem 1.1 and Corollary 1.2 can not be dropped entirely.

Example 3.4. Let $X_{0}=\left\{y_{i} \mid i \in \mathbf{Z}_{\geq 0}\right\}$ be a set and $X_{1}=\left\{z_{i} \mid i \in \mathbf{Z}_{\geq 0}\right\}$ a set. Define a poset $X=X_{0} \sqcup X_{1}$ with an order $\leq$ as follows: $y_{j} \leq z_{i}$ if and only if $i \leq j \in \mathbf{Z}_{\geq 0}$. Then $X$ is a non-spectral poset with diameter 2 . Indeed, for any elements $z_{i}, z_{j} \in X$ with $i<j, \downarrow z_{i} \cap \downarrow z_{j}=\left\{y_{k} \mid k \in \mathbf{Z}_{\geq j}\right\}$ and thus $\left|\downarrow z_{i} \cap \downarrow z_{j}\right|=\infty$. Since $\uparrow \downarrow z_{0}=X$, diam $X=2$. Since $\downarrow z_{i}$ are closed and $\bigcap_{i \geq 0} \downarrow z_{i}=\varnothing$, this implies that $\downarrow z_{0}$ is closed but not compact with respect to the upper topology. Thus $X$ is not compact with respect to the upper topology. Since any order compatible spectral topology contains the upper topology, there is no spectral topology on $X$.

\section{References}

[AZ04] M. E. Adams, van der Zypen, Representable posets and their order components, Cah. Topol. Géom. Différ. Catég. 45 (2004), 179-192.

[BE04] K. Belaid, O. Echi, On a conjecture about spectral sets, Topology Appl. 139 (2004), no. $1-3,1-15$.

[BF81] A. Bouvier, M. Fontana, Une classe d'espaces spectraux de dimension 1; les espaces principaux, Bull. Sci. Math. (2) 105 (1981), no. 2, 159-167.

[DFP80] D. E. Dobbs, M. Fontana, I. J. Papick, On certain distinguished spectral sets, Ann. Mat. Pura Appl., 128 (1980), 227-240.

[F79] M. Fontana, Sopra alcuni relativi agli insierni spettrali, Rend. Sem. Fac. Sci. Univ. Cagliari. 49 (1979), 445-467. 
[H69] M. Hochster, Prime ideal structure in commutative rings, Trans. Amer. Math. Soc. 142 (1969), 43-60.

[LO76] W. J. Lewis, J. Ohm, The ordering of $\operatorname{Spec}(\mathbf{R})$, Canad. J. Math., 28 (1976), pp. 820-835.

[P70] H. A. Priestley, Representation of distributive lattices by means of ordered Stone spaces, Bull. London Math. Sot. 2 (1970), 186-190.

[P94] H. A. Priestley, Intrinsic spectral topologies, Papers on general topology and applications (Flushing, NY, 1992), 78-95, Ann. New York Acad. Sci., 728, New York Acad. Sci., New York, 1994.

[S37] M. H. Stone, Topological representation of distributive lattices and Brouwerian logics, Casopis Pest. Mat. Fys. 67 (1937), 1-25.

[Y09] T. Yokoyama, A solution to a spectral poset problem of Lewis and Ohm, Topology Appl. 156 (2009), 2220-2223.

Faculty of Marine Technology

Tokyo University of Marine Science and Technology, 2-1-6, Etchujima

Koto-ku, Tokyo, 135-8533, Japan

E-mail address: yokoyama@ms.u-tokyo.ac.jp 